\title{
Coincidence and Common Fixed Point Theorems for Hybrid Mappings
}

\author{
Abdelkrim Aliouche and Valeriu Popa
}

\begin{abstract}
We prove common fixed point theorems for two pairs of hybrid mappings satisfying implicit relations in metric spaces using the concept of $T$-weak commutativity and we correct errors of [1], [4], [5] and [12]. Our Theorems generalize results of [1-5], [12] [16], [17-20] and $[26]$.
\end{abstract}

\section{Introduction and Preliminaries}

Let $(X, d)$ be a metric space. For $x \in X$ and $A \subset X, D(x, A)=$ $\inf \{d(x, y), y \in A\}$.

Let $C B(X)$ be the set of all nonempty closed and bounded subsets of $X$. Let $H$ be the Hausdorff metric with respect to $d$ defined by

$$
H(A, B)=\max \left\{\sup _{a \in A} D(a, B), \sup _{b \in B} D(A, b)\right\} \text { for all } A, B \in C B(X) .
$$

It is well known that $(C B(X), H)$ is a metric space and if $(X, d)$ is complete, then $(C B(X), H)$ is also complete

Lemma 1.1 ([14]). If $A, B \in C B(X)$ and $k>1$, then for each $a \in A$, there exists $b \in B$ such that $d(a, b) \leq k H(A, B)$.

Let $f: X \rightarrow X$ be a single-valued mapping and $T: X \rightarrow C B(X)$ be a multi-valued mapping.

Definition 1.2. 1) A point $x \in X$ is said to be a coincidence point of $f$ and $T$ if $f x \in T x$. We denote by $C(f, T)$ the set of all coincidence points of $f$ and $T$.

2) A point $x \in X$ is a fixed point of $T$ if $x \in T x$.

Definition 1.3. $\quad 1) \quad f$ and $T$ are said to be commuting [4] in $X$ if for all $x \in X, f T x \subset T f x$.

2000 Mathematics Subject Classification. Primary: 54H25, 47H10.

Key words and phrases. Hybrid mappings, Common fixed point, $T$-weakly commuting, metric space. 
2) $f$ and $T$ are said to be weakly commuting on $X[21,22]$ if for all $x \in X, f T x \in C B(X)$ and

$$
H(f T x, T f x) \leq D(f x, T x)
$$

3) $f$ and $T$ are said to be compatible $[7,11]$ if for all $x \in X, f T x \in$ $C B(X)$ and

$$
\lim _{n \rightarrow \infty} H\left(f T x_{n}, T f x_{n}\right)=0
$$

whenever $\left\{x_{n}\right\}$ is a sequence in $X$ such that $\lim _{n \rightarrow \infty} f x_{n}=t \in A=$ $\lim _{n \rightarrow \infty} T x_{n}$ for some $t \in X$ and $A \in C B(X)$.

Commuting implies weakly commuting implies compatible, but the converse is not true in general, see [9].

Definition 1.4. $\quad$ 1) $f$ and $T$ are said to be weakly compatible $[8,9]$ if they commute at their coincidence points; i.e., $f x \in T x$ implies that $f T x=T f x$.

2) $f$ and $T$ are said to be $R$-weakly commuting at $x \in X[15,23]$, if $f T x \in C B(X)$ and there exists an $R>0$ such that

$$
H(f T x, T f x) \leq R D(f x, T x)
$$

$f$ and $T$ are said to be pointwise $R$-weakly commuting on $X$ if for all $x \in X, f T x \in C B(X)$ and (1.1) holds for some $R>0$.

3) $f$ and $T$ are said to be (IT)-commuting at $x \in X[25]$ if $f T x \subset T f x$.

It is proved in [25] that a pointwise $R$-weakly commuting hybrid pair is not weakly compatible in general and $I T$-commutativity of $f$ and $T$ at a coincidence point is more general than their weak compatibility at the same point. However, pointwise $R$-weak commutativity at a coincidence point is equivalent to $(I T)$ commutativity at this point.

Definition 1.5 ([10]). $f$ is $T$-weakly commuting at $x \in X$ if $f f x \in T f x$.

Remark 1.6. $\quad 1)$ For a hybrid pair $(f, T),(I T)$ commuting at coincidence points implies that $f$ is $T$-weakly commuting at these points, but $T$-weakly commuting hybrid pair is neither IT-commuting nor compatible nor weakly commuting nor weakly compatible in general, see $[10]$.

2) If $T$ is a single-valued mapping, then $T$-weak commutativity at coincidence points is equivalent to weak compatibility of $f$ and $T$.

3) If $f$ and $T$ are single-valued maps then weak compatibility of $f$ and $T$ is equivalent to $R$-weak commutativity of $f$ and $T$ at their coincidence points.

Lemma 1.7. a) If $f$ is $T$-weakly commuting at $x \in X$, then $f x \in$ $C(f, T)$.

b) If $f$ is $T$-weakly commuting at $x \in X$ and $f x=f f x$, then $f x$ is a common fixed point of $f$ and $T$. 
The following Theorem was proved by [12].

Theorem 1.8. Let $(X, d)$ be a complete metric space, $T: X \rightarrow X$ and $F, G: X \rightarrow C B(X)$ satisfying

$$
F(X) \cup G(X) \subset T(X),
$$

$T$ is F-weakly commuting and

$T$ is $G$-weakly commuting at their coincidence points.

for all $x, y \in X, x \neq y, F x \neq F y$ and $G x \neq G y$, where $a, b>0$ and $a+2 b<1$, whenever $D(F x, T y)+D(G y, T x) \neq 0$ and $H(F x, G y)=0$ whenever $D(F x, T y)+D(G y, T x)=0$. Then, there exists $z \in X$ such that $z=T z \in F z \cap G z$.

In [17] and [18], the study of fixed points for mappings satisfying implicit relations was introduced and the study of a pair of hybrid mappings satisfying implicit relations was initiated in [19].

It is our purpose in this paper to prove coincidence and common fixed point theorems for two pairs of hybrid mappings satisfying implicit relations which generalize results of [1-5], [12], [16], [17-20] and [26].

\section{IMPLICIT RELATIONS}

Let $\Phi_{6}$ the family of all real continuous mappings $\phi\left(t_{1}, t_{2}, t_{3}, t_{4}, t_{5}, t_{6}\right)$ : $\mathbb{R}_{+}^{6} \rightarrow \mathbb{R}$ satisfying the following conditions:

$\left(\phi_{1}\right): \phi$ is increasing in variable $t_{1}$ and decreasing in variables $t_{3}, t_{4}, t_{5}$ and $t_{6}$.

$\left(\phi_{2}\right)$ : there exists $0 \leq h<1$ and $k>1$ such that

$\left(\phi_{a}\right): u \leq k t$ and $\phi(t, v, v, u, u+v, 0) \leq 0$ or

$\left(\phi_{b}\right): u \leq k t$ and $\phi(t, v, u, v, 0, u+v) \leq 0$ implies $u \leq h v$.

Example 2.1. $\phi\left(t_{1}, t_{2}, t_{3}, t_{4}, t_{5}, t_{6}\right)=t_{1}-a t_{2}-b\left(t_{3}+t_{4}\right)-c\left(t_{5}+t_{6}\right), a, c>0$, $b \geq 0$ and $a+2 b+2 c<1$.

$\left(\phi_{1}\right)$ : Obviously.

$\left(\phi_{2}\right):$ Let $1<k<\frac{1}{a+2 b+2 c}, u \leq k t$ and

$$
\phi(t, v, v, u, u+v, 0)=t-a v-b(v+u)-c(u+v) \leq 0 .
$$

Then, $u \leq k t \leq u \leq k a v+k b(v+u)+k c(u+v)]$ and so $u \leq h v$, where $h=\frac{k(a+b+c)}{1-(k b+k c)}<1$. Similarly, $u \leq k t$ and $\phi(t, v, u, v, 0, u+v) \leq 0$ implies $u \leq h v$. 
Example 2.2. $\phi\left(t_{1}, t_{2}, t_{3}, t_{4}, t_{5}, t_{6}\right)=t_{1}-a \max \left\{t_{2}, t_{3}, t_{4}, \frac{t_{5}+t_{6}}{2}\right\}$, $0<a<1$.

$\left(\phi_{1}\right)$ : Obviously.

$\left(\phi_{2}\right)$ : Let $1<k<\frac{1}{a}, u \leq k t$ and

$$
\phi(t, v, v, u, u+v, 0)=t-a \max \left\{v, u, \frac{u+v}{2}\right\} \leq 0 .
$$

Then,

$$
u \leq k t \leq k a \max \left\{v, u, \frac{u+v}{2}\right\} \leq k a \max \{v, u\} .
$$

If $u>0$ and $u \geq v$, it follows that $u \leq k a u<u$ which is a contradiction and so $u \leq h v$, where $h=k a<1$. If $u=0$, therefore $u \leq h v$. Similarly, $u \leq k t$ and $\phi(t, v, u, v, 0, u+v) \leq 0$ implies $u \leq h v$.

Example 2.3. $\phi\left(t_{1}, t_{2}, t_{3}, t_{4}, t_{5}, t_{6}\right)=t_{1}-a \max \left\{t_{2}^{2}, t_{3} t_{4}, t_{5} t_{6}, t_{3} t_{5}, t_{4} t_{6}\right\}^{\frac{1}{2}}$, $0<a<\frac{1}{\sqrt{2}}$.

$\left(\phi_{1}\right)$ : Obviously.

$\left(\phi_{2}\right):$ Let $1<k<\frac{1}{a \sqrt{2}}, u \leq k t$ and

$$
\phi(t, v, v, u, u+v, 0)=t-a \max \left\{v^{2}, u v, v(u+v)\right\}^{\frac{1}{2}} \leq 0 .
$$

Then,

$$
u \leq k t \leq k a \max \left\{v^{2}, u v, v(u+v)\right\}^{\frac{1}{2}} .
$$

If $u>0$ and $u \geq v$, it follows that $u \leq k a \sqrt{2} u<u$ which is a contradiction and so $u \leq h v$, where $h=k a \sqrt{2}<1$. If $u=0$, therefore $u \leq h v$. Similarly, $u \leq k t$ and $\phi(t, v, u, v, 0, u+v) \leq 0$ implies $u \leq h v$.

Example 2.4. $\phi\left(t_{1}, t_{2}, t_{3}, t_{4}, t_{5}, t_{6}\right)=t_{1}^{2}+\frac{t_{1}}{1+t_{5} t_{6}}-a t_{2}^{2}-b t_{3}^{2}-c t_{4}^{2}, a>0$, $b, c \geq 0$ and $a+b+c<1$.

$\left(\phi_{1}\right)$ : Obviously.

$\left(\phi_{2}\right):$ Let $1<k<\frac{1}{\sqrt{a+b+c}}, u \leq k t$ and

$$
\phi(t, v, v, u, u+v, 0)=t^{2}+t-a v^{2}-b v^{2}-c u^{2} \leq 0 .
$$

Then, $t^{2} \leq a v^{2}+b v^{2}+c u^{2}$ and $u^{2} \leq k^{2} t^{2} \leq k^{2}\left(a v^{2}+b v^{2}+c u^{2}\right)$. 
It follows that $u \leq h_{1} v$, where $h_{1}=k \sqrt{\frac{a+b}{1-k^{2} c}}<1$. Similarly, $u \leq k t$ and $\phi(t, v, u, v, 0, u+v) \leq 0$ implies $u \leq h_{2} v$, where $h_{2}=k \sqrt{\frac{a+c}{1-k^{2} b}}<1$. If $h=\max \left\{h_{1}, h_{2}\right\}$, then $u \leq h v$.

Example 2.5. $\phi\left(t_{1}, t_{2}, t_{3}, t_{4}, t_{5}, t_{6}\right)=$ $=t^{p}-\max \left\{a t_{2} t_{3}^{p-1}, a t_{2}^{p-1} t_{4}, a t_{3}^{p-1} t_{4}, c t_{5}^{p-1} t_{6}\right\}, p \geq 2,0<a<1$ and $c \geq 0$.

$\left(\phi_{1}\right)$ : Obviously.

$\left(\phi_{2}\right):$ Let $1<k<\frac{1}{\sqrt[p]{a}}, u \leq k t$ and

$$
\phi(t, v, v, u, u+v, 0)=t^{p}-\max \left\{a v^{p}, a v^{p-1} u\right\} \leq 0 .
$$

Then, $u^{p} \leq k^{p} t^{p} \leq k^{p} \max \left\{a v^{p}, a v^{p-1} u\right\}$. If $u>0$ and $u \geq v$, it follows that $u^{p} \leq a k^{p} u^{p}<u^{p}$ which is a contradiction and so $u \leq h v$, where $h=k \sqrt[p]{a}<1$. If $u=0$, therefore $u \leq h v$. Similarly, $u \leq k t$ and $\phi(t, v, u, v, 0, u+v) \leq 0$ implies $u \leq h v$.

Example 2.6. $\phi\left(t_{1}, t_{2}, t_{3}, t_{4}, t_{5}, t_{6}\right)=t_{1}-b\left[a \max \left\{t_{2}, t_{3}, t_{4}, \frac{t_{5}+t_{6}}{2}\right\}-\right.$ $\left.-(1-a) \max \left\{t_{2}^{2}, t_{3} t_{4}, t_{5} t_{6}, \frac{1}{2} t_{3} t_{6}, \frac{1}{2} t_{4} t_{5}\right\}^{\frac{1}{2}}\right], 0<b<1$ and $0 \leq a<1$.

$\left(\phi_{1}\right)$ : Obviously.

$\left(\phi_{2}\right)$ : Let $1<k<\frac{1}{b}, u \leq k t$ and

$$
\begin{aligned}
& \phi(t, v, v, u, u+v, 0)= \\
& =t-b\left[a \max \left\{v, u, \frac{u+v}{2}\right\}-(1-a) \max \left\{v^{2}, u v, \frac{1}{2} u(u+v)\right\}^{\frac{1}{2}}\right] \leq 0 .
\end{aligned}
$$

Then,

$$
u \leq k t \leq k b\left[a \max \left\{v, u, \frac{u+v}{2}\right\}+(1-a) \max \left\{v^{2}, u v, \frac{1}{2} u(u+v)\right\}^{\frac{1}{2}}\right] .
$$

If $u>0$ and $u \geq v$, it follows that $u \leq k b u<u$ which is a contradiction and so $u \leq h v$, where $h=k b<1$. If $u=0$, therefore $u \leq h v$. Similarly, $u \leq k t$ and $\phi(t, v, u, v, 0, u+v) \leq 0$ implies $u \leq h v$.

Example 2.7. $\phi\left(t_{1}, t_{2}, t_{3}, t_{4}, t_{5}, t_{6}\right)=t_{1}-a t_{2}-b \frac{t_{5}^{2}+t_{6}^{2}}{t_{5}+t_{6}}-c\left(t_{3}+t_{4}\right)$, $t_{5}+t_{6} \neq 0, a, b>0, c \geq 0$ and $a+2 b+2 c<1$.

Example 2.8. $\phi\left(t_{1}, t_{2}, t_{3}, t_{4}, t_{5}, t_{6}\right)=t_{1}-a t_{2}-b \frac{t_{3}^{2}+t_{4}^{2}}{t_{3}+t_{4}}-c\left(t_{5}+t_{6}\right)$, $t_{3}+t_{4} \neq 0, a, b, c>0$ and $a+2 b+2 c<1$. 
They follow as in Example 2.1 since $\frac{t_{5}^{2}+t_{6}^{2}}{t_{5}+t_{6}} \leq t_{5}+t_{6}$ and $\frac{t_{3}^{2}+t_{4}^{2}}{t_{3}+t_{4}} \leq t_{3}+t_{4}$ if $t_{5}+t_{6} \neq 0$ and $t_{3}+t_{4} \neq 0$.

\section{Main Results}

Theorem 3.1. Let $(X, d)$ be a metric space, $S, T: X \rightarrow X$ and $F, G: X \rightarrow$ $C B(X)$ satisfying

$$
\begin{gathered}
F(X) \subset T(X) \text { and } G(X) \subset S(X) \\
\phi(H(F x, G y), d(S x, T y), D(S x, F x), D(T y, G y), \\
D(S x, G y), D(F x, T y)) \leq 0
\end{gathered}
$$

for all $x, y \in X$, where $\phi \in \Phi_{6}$, whenever $D(S x, G y)+D(F x, T y) \neq 0$ and $H(F x, G y)=0$ whenever $D(S x, G y)+D(F x, T y)=0$. Suppose that one of $S(X)$ or $T(X)$ is complete. Then

a) there exists $p, q \in X$ such that $S p \in F p$ and $T q \in G q$.

Further, if $S$ is F-weakly commuting and $T$ is $G$-weakly commuting at their coincidence points, therefore

b) There exists $z \in X$ such that $S z \in F z$ and $T z \in G z$.

c) In the case (b), if $S z=T z$, then $S z=T z \in F z \cap G z$.

d) In the case (c), if $S z=T z=z$, then $z$ is a common fixed point of $S, T, F$ and $G$.

Proof. First, assume that there exists $p, q \in X$ such that $D(S p, G q)+$ $D(F p, T q)=0$. So, $D(S p, G q)=0$ and $D(F p, T q)=0$ which implies that $S p \in G q$ and $T q \in F p$. Since $H(F p, G q)=0$, it follows that $D(S p, F p) \leq$ $H(F p, G q)=0$ and hence $S p \in F p$. In a similar manner, we get $T q \in G q$.

Now, assume that $D(S x, G y)+D(F x, T y) \neq 0$ for all $x, y \in X$. Let $x_{0} \in X$ be an arbitrary point. By (3.1) and Lemma 1.1, we define a sequence $\left\{y_{n}\right\}$ in $X$ such that

$$
y_{2 n}=S x_{2 n} \in G x_{2 n-1}, \quad y_{2 n+1}=T x_{2 n+1} \in F x_{2 n}
$$

and

$$
\begin{aligned}
d\left(y_{2 n}, y_{2 n+1}\right) & \leq k H\left(F x_{2 n}, G x_{2 n-1}\right), \\
d\left(y_{2 n+1}, y_{2 n+2}\right) & \leq k H\left(F x_{2 n}, G x_{2 n+1}\right), \quad \text { for } \quad n=1,2, \ldots
\end{aligned}
$$


Using (3.2) and $\left(\phi_{1}\right.$, we have

$$
\begin{gathered}
0 \geq \phi\left(H\left(F x_{2 n}, G x_{2 n-1}\right), d\left(S x_{2 n}, T x_{2 n-1}\right), D\left(S x_{2 n}, F x_{2 n}\right),\right. \\
\left.D\left(T x_{2 n-1}, G x_{2 n-1}\right), D\left(S x_{2 n}, G x_{2 n-1}\right), D\left(F x_{2 n}, T x_{2 n-1}\right)\right) \\
\geq \phi\left(H\left(F x_{2 n}, G x_{2 n-1}\right), d\left(y_{2 n-1}, y_{2 n}\right), d\left(y_{2 n}, y_{2 n+1}\right),\right. \\
\left.d\left(y_{2 n-1}, y_{2 n}\right), 0, d\left(y_{2 n-1}, y_{2 n+1}\right)\right) . \\
\geq \phi\left(H\left(F x_{2 n}, G x_{2 n-1}\right), d\left(y_{2 n-1}, y_{2 n}\right), d\left(y_{2 n}, y_{2 n+1}\right),\right. \\
\left.d\left(y_{2 n-1}, y_{2 n}\right), 0, d\left(y_{2 n-1}, y_{2 n}\right)+d\left(y_{2 n}, y_{2 n+1}\right)\right) .
\end{gathered}
$$

By $\left(\phi_{b}\right)$, we obtain

$$
d\left(y_{2 n}, y_{2 n+1}\right) \leq h d\left(y_{2 n-1}, y_{2 n}\right)
$$

In the same manner, applying (3.3) we get

$$
\begin{gathered}
0 \geq \phi\left(H\left(F x_{2 n}, G x_{2 n+1}\right), d\left(S x_{2 n}, T x_{2 n+1}\right), D\left(S x_{2 n}, F x_{2 n}\right),\right. \\
\left.D\left(T x_{2 n+1}, G x_{2 n+1}\right), D\left(S x_{2 n}, G x_{2 n+1}\right), D\left(F x_{2 n}, T x_{2 n+1}\right)\right) \\
\geq \phi\left(H\left(F x_{2 n}, G x_{2 n+1}\right), d\left(y_{2 n}, y_{2 n+1}\right), d\left(y_{2 n}, y_{2 n+1}\right),\right. \\
\left.d\left(y_{2 n+1}, y_{2 n+2}\right), d\left(y_{2 n}, y_{2 n+1}\right)+d\left(y_{2 n+1}, y_{2 n+2}\right), 0\right) .
\end{gathered}
$$

By $\left(\phi_{a}\right.$, we obtain

$$
d\left(y_{2 n+1}, y_{2 n+2}\right) \leq h d\left(y_{2 n}, y_{2 n+1}\right)
$$

and hence

$$
d\left(y_{n}, y_{n+1}\right) \leq h d\left(y_{n-1}, y_{n}\right)
$$

Therefore, $\left\{y_{n}\right\}$ is a Cauchy sequence in $X$. As $S(X)$ is complete, it converges to $z \in S(X)$ and so there exists $p \in X$ such that $z=S p$. Using (3.2) and $\left(\phi_{1}\right)$ we have

$$
\begin{gathered}
0 \geq \phi\left(H\left(F p, G x_{2 n-1}\right), d\left(S p, T x_{2 n-1}\right), D(S p, F p)\right. \\
\left.D\left(T x_{2 n-1}, G x_{2 n-1}\right), D\left(S p, G x_{2 n-1}\right), D\left(F p, T x_{2 n-1}\right)\right) \\
\geq \phi\left(D\left(F p, y_{2 n}\right), d\left(S p, y_{2 n-1}\right), D(S p, F p),\right. \\
\left.d\left(y_{2 n-1}, y_{2 n}\right), d\left(S p, y_{2 n}\right), D\left(y_{2 n-1}, F p\right)\right) .
\end{gathered}
$$

Letting $n$ tend to infinity, we get

$$
\phi(D(F p, S p), 0, D(F p, S p), 0,0, D(F p, S p)) \leq 0 .
$$


By $\left(\phi_{b}\right)$ we obtain $S p \in F p$. Similarly, as $F(X) \subset T(X)$, there exists $q \in X$ such that $z=S p=T q$. Applying (3.2) and $\left(\phi_{1}\right)$ we have

$$
\begin{gathered}
0 \geq \phi\left(H\left(F x_{2 n}, G q\right), d\left(S x_{2 n}, T q\right), D\left(S x_{2 n}, F x_{2 n}\right),\right. \\
\left.D(T q, G q), D\left(S x_{2 n}, G q\right), D\left(F x_{2 n}, T q\right)\right) \\
\geq \phi\left(D\left(y_{2 n+1}, G q\right), d\left(y_{2 n}, T q\right), d\left(y_{2 n}, y_{2 n+1}\right),\right. \\
\left.D(T q, G q), D\left(y_{2 n}, G q\right), d\left(y_{2 n+1}, T q\right)\right)
\end{gathered}
$$

Letting $n$ tend to infinity, we get

$$
\phi(D(T q, G q), 0,0, D(T q, G q), D(T q, F q), 0) \leq 0 .
$$

By $\left(\phi_{a}\right)$ we obtain $T q \in G q$. Since $S$ is $F$-weakly commuting at $p \in C(S, T)$ and $T$ is $G$-weakly commuting at $q \in C(G, T)$ it follows by Lemma 1.7 (a) that $z=S p \in C(F, T)$ and $z=T q \in C(G, T)$. Hence, $S z \in F z$ and $T z \in G z$. If $S z=T z$, then $S z=T z \in F z \cap G z$ and if $S z=T z=z$, then $z$ is a common fixed point of $S, T, F$ and $G$.

Corollary 3.2. Let $(X, d)$ be a metric space, $S, T: X \rightarrow X$ and $F, G$ : $X \rightarrow C B(X)$ satisfying (3.1) and

$H(F x, G y) \leq a d(S x, T y)+b(D(S x, F x)+D(T y, G y))+c(D(S x, G y), D(F x, T y))$

for all $x, y \in X$, where $a, c>0, b \geq 0$ and $a+2 b+2 c<1$. Suppose that one of $S(X)$ or $T(X)$ is complete. Then, the conclusions (a), (b), (c) and (d) of Theorem 3.1 hold.

Proof. It follows from Theorem 3.1 and Example 2.1.

Remark 3.3. In Theorems of [1] and [12], to prove that $z=T z$, the authors used: " $T x_{2 n} \in G x_{2 n-1}$ and $T z \in F z$ implies that $d\left(T x_{2 n}, T z\right) \leq$ $H\left(G x_{2 n-1}, F z\right)$ " which is false because " $a \in A$ and $b \in B$ implies $d(a, b) \leq$ $H(A, B)$ " is not true in general as it shown by the following example.

Example 3.4. Let $d(x, y)=|x-y|, A=\left[0, \frac{1}{2}\right]$ and $B=\left[\frac{1}{4}, 1\right]$. We have $0 \in A$ and $1 \in B$, but $d(0,1)=1>H(A, B)=\frac{1}{2}$. Therefore, Theorem 1.8 of [12] is false as it is proved by the following Example.

Example 3.5. Let $(X, d)=([1, \infty),||),. S x=T x=x^{2}+1$ and $F x=G x=$ $[2, x+3]$ for all $x \in X$. It is easy to verify that for all $x, y \in X$

$$
\begin{aligned}
d(S x, S y) & =\left|x^{2}-y^{2}\right| \\
& \geq 2|x-y| \\
& =H(F x, F y)
\end{aligned}
$$


and hence

$$
\begin{aligned}
H(F x, F y) & \leq \frac{1}{2} d(S x, S y) \\
& \leq \frac{1}{2} d(S x, S y)+\frac{1}{8} \frac{D^{2}(F x, S y)+D^{2}(S x, F y)}{D(F x, S y)+D(S x, F y)} .
\end{aligned}
$$

It is easy to see that the other conditions of Theorem 1.8 of [12] are satisfied, but $S$ and $F$ have no common fixed point.

The following Corollary is the correct form of Theorem 1.8 of [12].

Corollary 3.6. Let $(X, d)$ be a complete metric space, $T: X \rightarrow X$ and $F, G: X \rightarrow C B(X)$ satisfying (1.2) and

$$
H(F x, G y) \leq a d(T x, T y)+c \frac{D^{2}(F x, T y)+D^{2}(T x, G y)}{D(F x, T y)+D(T x, G y)}
$$

for all $x, y \in X$, where $a, c>0$ and $a+2 c<1$, whenever $D(T x, G y)+$ $D(F x, T y) \neq 0$ and $H(F x, G y)=0$ whenever $D(T x, G y)+D(F x, T y)=0$. Then, (a) holds. Further, if $T$ is $F$-weakly commuting and $T$ is $G$-weakly commuting at their coincidence points, therefore the conclusions (b), (c) and (d) of Theorem 3.1 hold.

Proof. It follows from The fact that

$$
\frac{D^{2}(F x, T y)+D^{2}(T x, G y)}{D(F x, T y)+D(T x, G y)} \leq D(F x, T y)+D(T x, G y)
$$

if $D(T x, G y)+D(F x, T y) \neq 0$ and Corollary 3.2.

Remark 3.7. In [17] Remark 3 and [12] Remark 5, we have: "the conditions in the hypothesis of Theorem 3.1 of [1] and Theorem 1 of [12], $x \neq y, F x \neq$ $F y$ and $G x \neq G y$ are necessary since the Theorem fails for $F$ and $G$ taken as constant mappings". This is demonstrated by the following example.

Example 3.8. Let $X=\{0,1\}, T x=1-x$ and $F x=G x=\{0,1\}$ for all $x \in X$. It is easy to verify that the mappings satisfy all the hypothesis except $x \neq y, F x \neq F y$.

Remark 3.9. 1) In Example 3.8, we have $T(0) \in F(0)$ and $T(1) \in$ $F(1)$; i.e., $T$ and $F$ have coincidence points. Since $T^{2}(0) \neq T(0)$ and $T^{2}(1) \neq T(1), T$ and $F$ have no common fixed point.

2) In Theorems of [1], [4] and [12], $x \neq y, F x \neq F y$ and $G x \neq G y$ are not necessary as it is shown by the following Example.

3) In Theorem 1 of [26], $S$ and $g$ are compatible should be the pairs $(S, f)$ and $(T, G)$ are compatible and in Corollary $2, g$ should be replaced by $f$ and the pair $(S, f)$ is compatible should be added.

4) In the paper of Imdad and J. Ali [5], the condition $\left(\phi_{b}\right)$ should be added in $\left(G_{2}\right)$ in order to prove that $d\left(y_{2 n}, y_{2 n+1}\right) \leq h d\left(y_{2 n-1}, y_{2 n}\right)$ 
and the condition $\left(G_{3}\right)$ should be deleted because it can be deduced by $\phi_{b}$ for $v=0$.

5) In [16], the authors made the following remark . It is not yet known whether their Theorem remains true if one of the mappings $f$ and $T$ is not continuous and Theorem 2 of [25] yields that the answer is affirmative.

Example 3.10. Let $X=\left\{0,1, \frac{1}{2}\right\}, T x=1-x$ and $F x=G x=\left\{0, \frac{1}{2}, 1\right\}$ for all $x \in X$. It is easy to verify that the mappings satisfy the conditions of Theorems of [1], [4] and [12] except $x \neq y, F x \neq F y$, but $T\left(\frac{1}{2}\right)=\frac{1}{2} \in F\left(\frac{1}{2}\right)$ and so $\frac{1}{2}$ is a common fixed point of $T$ and $F$.

As $x \neq y, F x \neq F y$ and $G x \neq G y$ are not necessary, it follows that Theorem of [1] and Theorems 3.2 and 3.3 of [4] part (a) are false, it suffices to take Example 3.8 for [1] and $X=\{0,1\}, T x=1-x, S x=I x=J x=x$ and $F x=G x=\{0,1\}$ for all $x \in X$ for [4].

We can also prove the following Theorem which generalizes Theorems 3.2 and 3.3 of [4].

Theorem 3.11. Let $(X, d)$ be a metric space, $S, T: X \rightarrow X$ and $F, G$ : $X \rightarrow C B(X)$ satisfying

$$
\begin{gathered}
F(X) \subset T g(X) \text { and } G(X) \subset S f(X) \\
\phi(H(F x, G y), d(S f x, T g y), D(S f x, F x), D(T g y, G y), \\
D(S f x, G y), D(F x, T g y)) \leq 0
\end{gathered}
$$

for all $x, y \in X$, where $\phi \in \Phi_{6}$, whenever $D(S f x, G y)+D(F x, T g y) \neq 0$ and $H(F x, G y)=0$ whenever $D(S f x, G y)+D(F x, T g y)=0$. Suppose that one of $S(X)$ or $T(X)$ is complete. Then

a) There exists $p, q \in X$ such that $S f p \in F p$ and $T g q \in G q$.

Further, if $S f$ is F-weakly commuting and $T g$ is $G$-weakly commuting at their coincidence points, therefore

b) There exists $z \in X$ such that $S f z \in F z$ and $T g z \in G z$.

c) In the case (b), if $S f z=T g z$, then $S f z=T g z \in F z \cap G z$.

d) In the case (c), if $S f z=T g z=z,(S, f),(S f, S),(T, g),(T g, T)$ commute, $S^{2} z=S z, f^{2} z=f z, T^{2} z=T z$ and $g^{2} z=g z$, then $z$ is a common fixed point of $f, S, T, g, S f, T g, F$ and $G$.

The following Theorem generalizes Theorems of Popa [17-20] and Imdad et al [3].

Theorem 3.12. Let $(X, d)$ be a metric space, $S, T: X \rightarrow X$ and $F, G$ : $X \rightarrow C B(X)$ satisfying (3.1) and

$\phi(H(F x, G y), d(S x, T y), D(S x, F x), D(T y, G y), D(S x, G y), D(F x, T y)) \leq 0$ 
for all $x, y \in X$, where $\phi \in \Phi_{6}$. Suppose that one of $S(X)$ or $T(X)$ is complete. Then, (a) holds. Further, if $S$ is F-weakly commuting and $T$ is $G$-weakly commuting at their coincidence points, therefore the conclusions (b), (c) and (d) of Theorem 3.1 hold.

In the same manner, we can prove the following Theorem which extend and improve Theorem 3.1 of Imdad and Ali [5].

Theorem 3.13. Let $\left\{F_{n}\right\}_{n>1}$ be a sequence of multi-valued mappings from a metric space $(X, d)$ into $C B(X)$ and $S, T: X \rightarrow X$ satisfying

$$
\begin{gathered}
F_{i}(X) \subset T(X) \text { and } F_{j}(X) \subset S(X) \\
\phi\left(H\left(F_{i} x, F_{j} y\right), d(S x, T y), D\left(S x, F_{i} x\right), D\left(T y, F_{j} y\right),\right. \\
\left.D\left(S x, F_{j} y\right), D\left(F_{i} x, T y\right)\right) \leq 0
\end{gathered}
$$

for all $x, y \in X$, where $\phi \in \Phi_{6}$ and $i=2 n-1, j=2 n, n \geq 1$. Suppose that one of $S(X)$ or $T(X)$ is complete. Then

a) There exists $p, q \in X$ such that $S p \in F_{i} p$ and $T q \in F_{j} q$.

Further, if $S$ is $F_{i}$-weakly commuting and $T$ is $F_{j}$-weakly commuting at their coincidence points, therefore

b) There exists $z \in X$ such that $S z \in F_{i} z$ and $T z \in F_{j} z$.

c) In the case (b), if $S z=T z$, then $S z=T z \in F_{i} z \cap F_{j} z$.

d) In the case (c), if $S z=T z=z$, then $z$ is a common fixed point of $S, T, F_{i}$ and $G_{j}$.

Theorem 3.14. Let $\left\{F_{n}\right\}_{n \geq 1}$ be a sequence of mappings from a metric space $(X, d)$ into $C B(X)$ and $S, T: X \rightarrow X$ satisfying

$$
\begin{array}{r}
F_{1}(X) \subset T(X) \quad \text { and } \quad F_{n}(X) \subset S(X), \quad n>1 \\
\phi\left(H\left(F_{1} x, F_{n} y\right), d(S x, T y), D\left(S x, F_{1} x\right), D\left(T y, F_{n} y\right),\right. \\
\left.D\left(S x, F_{n} y\right), D\left(F_{1} x, T y\right)\right) \leq 0
\end{array}
$$

for all $x, y \in X$, where $\phi \in \Phi_{6}$, whenever $D\left(S x, F_{i} y\right)+D\left(F_{1} x, T y\right) \neq 0$ and $H(F x, G y)=0$ whenever $D\left(S x, F_{i} y\right)+D\left(F_{1} x, T y\right)=0$. Suppose that one of $S(X)$ or $T(X)$ is complete. Then

a) There exists $p, q \in X$ such that $S p \in F_{1} p$ and $T q \in F_{n} q, n>1$.

Further, if $S$ is $F_{1}$-weakly commuting and $T$ is $F_{n}$-weakly commuting at their coincidence points for $n>1$, therefore

b) There exists $z \in X$ such that $S z \in F_{1} z$ and $T z \in F_{i} z$.

c) In the case (b), if $S z=T z$, then $S z=T z \in F_{1} z \cap F_{i} z$.

d) In the case (c), if $S z=T z=z$, then $z$ is a common fixed point of $T_{n}, F$ and $G$. 
The following Theorem generalizes Theorems of Popa [17-20], Imdad et al [3] and Djoudi and Aliouche [2].

Theorem 3.15. Let $\left\{F_{n}\right\}_{n \geq 1}$ be a sequence of multi-mappings from a metric space $(X, d)$ into $C B(X)$ and $S, T: X \rightarrow X$ satisfying

$$
\begin{array}{r}
F_{1}(X) \subset T(X) \quad \text { and } \quad F_{n}(X) \subset S(X), \quad n>1 \\
\phi\left(H\left(F_{1} x, F_{n} y\right), d(S x, T y), D\left(S x, F_{1} x\right), D\left(T y, F_{n} y\right),\right. \\
\left.D\left(S x, F_{n} y\right), D\left(F_{1} x, T y\right)\right) \leq 0
\end{array}
$$

for all $x, y \in X$, where $\phi \in \Phi_{6}$. Suppose that one of $S(X)$ or $T(X)$ is complete. Then, (a) holds. Further, if $S$ is $F_{1}$-weakly commuting and $T$ is $F_{n}$-weakly commuting at their coincidence points for $n>1$, therefore, the conclusions (b), (c) and (d) of Theorem 3.14 hold.

Acknowledgements. The authors would like to thank Dr. Ali for his paper [5].

\section{REFERENCES}

[1] A.J. Asad and Z. Ahmad, A common fixed point of multi-valued mappings with weak commutativity conditions, Radovi Math. 9 (1999), 119-124.

[2] A. Djoudi and A. Aliouche, A general common fixed point theorem for reciprocally continuous mappings satisfying an implicit relation, The Austral. J. Math. Anal. Appl., 3 (2006), 1-7.

[3] M. Imdad, S. Kumar and M. S. Khan, Remarks on some fixed point theorems satisfying implicit relations, Radovi Mat., 1 (2002), 35-143.

[4] M. Imdad and T. I. Khan, Results on Nonlinear Hybrid Contractions Satisfying a Rational Inequality, Southeast Asian Bulletin of Mathematics., 26 (2002), 421-432.

[5] M. Imdad and J. Ali, A General Fixed Point Theorem for Hybrid Contractions via Implicit Functions, Southeast Asian Bulletin of Mathematics., 31 (2007) 73-80.

[6] S. Itoh and W. Takahashi, Single-valued mappings, Multi-valued mappings and fixed point theorems, J. Math. Anal. Appl., 59 (1977), 514-521.

[7] G. Jungck, Compatible mappings and common fixed points, Int. J. Math. Math. Sci., 9 (1986), 771-779.

[8] G. Jungck, Common fixed points for non-continuous non-self maps on non metric spaces, Far East J. Math. Sci., 4 (2) (1996), 199-215.

[9] G. Jungck and B.E. Rhoades, Fixed points for set-valued functions without continuity, Indian J. Pure Appl. Math., 29 (3) (1998), 227-238.

[10] T. Kamran, Coincidence and fixed points for hybrid strict contractions, J. Math. Anal. Appl., 299 (2004), 253-241.

[11] H. Kaneko and S. Sessa, Fixed point theorems for compatible multi-valued and single valued mappings, Internat. J. Math. Math. Sci., 12 (1989), 257-262.

[12] Kubiaczyk and Bhavana Deshpande, A Common Fixed Point Theorem for Multivalued Mappings Through T-weak Commutativity, Mathematica Moravica., 10 (2006), 55-60. 
[13] W. Liu, J. Wu and Z. Li, Common fixed points of single-valued and multi-valued maps, Internat. J. Math. Math. Sci., 19 (2005), 3045-3055.

[14] S.B. Nadler, Multi-valued contractions mappings, Pacific J. Math., 30 (2) (1969), 475-488.

[15] R.P. Pant, Common fixed points of noncommuting mappings, J. Math. Anal. Appl., 188 (1994), 436-440.

[16] H.K. Pathak and M. S. Khan, Fixed and coincidence points of hybrid mappings, Arch. Math. (Brno), 38 (3) (2002), 201-208.

[17] V. Popa, Some fixed point theorems for contractive mappings, Stud. Cer. St. Ser. Mat. Univ. Bacau, 7 (1997), 157-163.

[18] V. Popa, Some fixed point theorems for compatible mappings satisfying an implicit relation, Demonsratio Math., 32 (1) (1999), 157-163.

[19] V. Popa, A general coincidence theorem for compatible multi-valued mappings satisfying an implicit relation, Demonstratio Math., 33 (1) (2000), 159-164.

[20] V. Popa, Coincidence and fixed points theorems for noncontinuous hybrid contractions, Nonlinear Anal. Forum., 7 (2) (2002), 153-158.

[21] S. Sessa, On a weak commutativity condition of mappings in fixed point considerations, Publ. Inst. Math., (Beograd), 32 (46) (1982), 149-153.

[22] S. Sessa, M.S. Khan and M. Imdad, A common fixed point theorem with a weak commutativity condition, Glas. Math. Ser., III 21 (1986), 225-235.

[23] N. Shahzad and T. Kamran, Coincidence points and R-weakly commuting maps, Arch. Math. (Brno) 37 (2001), 179-183.

[24] S.L. Singh and S.N. Mishra, Some remarks on coincidences and fixed points, C. R. Math. Rep. Acad. Sci. Canada., 18 2-3 (1996), 66-70.

[25] S.L. Singh and S. N. Mishra, Coincidence and fixed points of non-self hybrid contractions, J. Math. Anal. Appl., 256 (2001), 486-497.

[26] D. Turkoglu, O. Ozer and B. Fisher, A coincidence point theorem for multi-valued contractions, Math. Communications., 7 (2002), 39-44.

Abdelkrim Aliouche
Department of Mathematics
University of LARBi Ben M'Hidi
Oum-El-BOuAGHi, 04000
AlgERIA
E-mail address: alioumath@yahoo.fr

VALERIU POPA

DePartment of Mathematics

UNIVERSITY OF BACĂU

STR. SPIRU HARET NR. 8

600114 BACĂU

ROMANIA

E-mail address: vpopa@ub.ro 